\title{
Analítica de datos aplicada a la caracterización microbiológica y sensorial de miel de abejas proveniente del departamento de Sucre, Colombia
}

\author{
Data Analytics Applied to the Microbiological and Sensory Characterization of Honey from the \\ Department of Sucre, Colombia
}

\begin{abstract}
Andrea Menco Tovar ${ }^{a d}$, María Méndez Ramos ${ }^{b e}$, Kettis Cáceres Pestana ${ }^{c f}$, Melba Vertel Morinson ${ }^{a g}$
${ }^{a}$ Grupo de Investigación Estadística y Modelamiento Matemático Aplicado a Calidad Educativa (GEMMA), Departamento de Matemáticas, Facultad de Educación y Ciencias, Universidad de Sucre, Colombia

${ }^{\mathrm{b}}$ Grupo de Investigación Estadística y Modelamiento Matemático Aplicado a Calidad Educativa (GEMMA), Departamento de Economía, Facultad Ciencias Económicas y Administrativas, Universidad de Sucre, Colombia

' Departamento de Economía, Facultad Ciencias Económicas y Administrativas, Universidad de Sucre, Colombia

${ }^{\mathrm{d}}$ https://orcid.org/0000-0002-6861-7547

${ }^{\mathrm{e}}$ https://orcid.org/0000-0002-7477-4717

${ }^{f}$ https://orcid.org/0000-0002-9782-190X

g melba.vertel@unisucre.edu.co | https://orcid.org/0000-0002-3204-5145
\end{abstract}

Citation: Menco-Tovar, A., MéndezRamos, M., Cáceres-Pestana, K. y VertelMorinson, M. (2022). Analítica de datos aplicada a la caracterización microbiológica y sensorial de miel de abejas del departamento de Sucre, Colombia. Mutis, 12(1). https://doi.org/10.21789/22561498.1768

Recibido: 9 de junio de 2021

Aceptado: 30 de septiembre de 2021

Copyright: (C) 2021 por los autores. Licenciado para Mutis. Este artículo es un artículo de acceso abierto distribuido bajo los términos y condiciones de la licencia Creative Commons Attribution (https:// https://creativecommons.org/licenses/bync-sa/4.0/).

\section{RESUMEN}

La presente investigación tiene como propósito caracterizar con la mayor precisión posible los factores microbiológicos y sensoriales de la miel de abejas producida y comercializada por una empresa de la cadena apícola del departamento de Sucre, mediante la utilización de herramientas y técnicas de analítica de datos, además del uso de software estadístico de acceso libre, a fin de contribuir a la formación de competencias investigativas, científicas y tecnológicas para el fortalecimiento del sector productivo en la región. Se realizó la revisión bibliográfica de las características teóricas de la analítica de datos. La información recolectada en los análisis microbiológicos y sensoriales fue codificada, tabulada y analizada para evaluar la composición de las muestras de la miel de abejas; los datos obtenidos para todas las variables cuantificadas se sometieron a técnicas multivariadas, tales como el análisis de correspondencia múltiple. En el comportamiento de las variables sensoriales de las muestras de miel, se evidencia que las que más contribuyen a las dimensiones que conforman el análisis de correspondencia múltiple son el color, el sabor y la textura. Para el caso del factor microbiológico, el recuento de esporas, levaduras y mohos en las muestras permite evidenciar un nivel igual para todos los lotes incluidos en el análisis. Las muestras involucradas en el estudio presentan buen comportamiento en términos de los indicadores microbiológicos evaluados.

Palabras clave: miel, microbiológico, sensorial, caracterización, análisis multivariado, contaminantes, organoléptico, parámetros, prevalencia

\section{ABSTRACT}

The purpose of this research is to characterize with the greatest possible precision the microbiological and sensory factors of the honey produced and commercialized by one company in the apiculture chain of the department of Sucre, 
through the use of analytical tools and data techniques, in addition to the use of free access statistical software, aiming to contribute to the formation of research, scientific and technological competences for strengthening the productive sector in this region. A bibliographic review of the theoretical characteristics of the data analysis was carried out; the information collected in the microbiological and sensory analyzes was coded, tabulated and analyzed to evaluate the composition of the samples of honey; the data obtained for all the quantified variables were subjected to multivariate techniques such as multiple correspondence analysis. In the behavior of the sensory variables of honey samples, it is evident that those that most take advantage of the dimensions of multiple correspondence analysis are color, flavor and texture. In the case of the microbiological factor, the count of spores, yeasts and molds in the samples shows an equal level for all the batches included in the analysis. The samples involved in the study show good performance in terms of the microbiological indicators evaluated.

Keywords: Honey, microbiological, sensory, characterization, multivariate analysis, contaminants, organoleptic, parameters, prevalence

\section{INTRODUCCIÓN}

La analítica de datos (o data analytics) es la ciencia que se basa en la utilización de información de una empresa, institución u organización en cualquier formato (preferiblemente digital), con el propósito de extraer conocimientos que ayuden a los implicados a tomar mejores decisiones a corto, mediano y largo plazo, lo cual también les permitirá conocer el estado actual en el que se encuentran, además de que proporciona un camino de respuestas a las múltiples inquietudes que se puedan presentar (Joyanes, 2014; Lugo \& López, 2018; Morales et al., 2019; Ordieres, 2014; Ortiz et al., 2015; Tascón, 2013).

A nivel empresarial existe constantemente la necesidad (creciente en estos tiempos) de analizar gran cantidad de información generada a partir de las actividades diarias y proveniente de diversas fuentes. Debido a lo anterior, este proceso requiere de un tratamiento apropiado de los datos, que permita a las organizaciones identificar problemas e inconsistencias de una forma más comprensible, detallada y precisa, para así finalmente eliminar las áreas problemáticas de manera rápida y eficiente, aprovechando al máximo sus fortalezas, lo cual conduce a mayores ganancias, beneficios y clientes satisfechos (Aristizábal, 2017; Gómez, 2019; Lugo \& López, 2018; Pastor, 2017). En el ámbito empresarial, aunque pareciera algo exclusivo de grandes empresas, las pequeñas y medianas organizaciones también pueden hacer uso de herramientas de analítica de datos, ya que el uso de estas puede conllevar a movimientos de negocios más inteligentes, rápidos y sin problemas, con mejores ganancias, mayores beneficios y operaciones más eficientes, lo cual ofrece la posibilidad de mejorar la cadena productiva y su actividad económica.

A pesar del auge de la analítica de datos en Colombia, más del $60 \%$ de las empresas aún no realiza procesos de innovación ni apropiación tecnológica que les permitan ser más eficaces, rentables y competentes, así como reducir costos de producción y comercialización. Asimismo, las múltiples variables que afectan al sector agro en la región, debido a la competencia en el mercado y a los diversos agentes que intervienen en la producción debido al cambio climático, empiezan a hacer evidente la necesidad de enfatizar en la toma de decisiones y estrategias de negocios apoyada en los datos y la información (FAO, 2014). 
Dentro del sector agro se resalta la producción de miel de abejas, sustancia natural producida por la abeja melífera (Apis mellifera) y otras especies, las cuales toman de las flores el néctar y otras secreciones que absorben, transportan, transforman y almacenan en panales (Dardón \& Enríquez, 2008).

Fabio Diazgranados, presidente de Fedeabejas, afirma que en Colombia el $90 \%$ de los apicultores tiene apiarios tipo, es decir, aquellos que poseen en promedio 20 colmenas. También, considera que en el país no existe apicultura intensiva, ya que son contados los grandes apicultores con más de 500 colmenas. Sin embargo, la apicultura colombiana es una actividad económica agroindustrial en consolidación dentro de una dinámica de mercado creciente, calculándose el promedio anual nacional en unos $32 \mathrm{~kg}$ de miel por colmena, con una producción de 3.112 toneladas en 2015. En términos regionales, la mayor cantidad de colmenas se ubica en las regiones Andina y Caribe, en las cuales se destacan departamentos como Huila, Sucre, Bolívar y Córdoba, tradicionalmente reconocidos por la producción de miel de abejas (González \& Echenique, 2017; Sánchez et al., 2013).

En el caso del departamento de Sucre, a pesar de la presencia de un gran número de apicultores, de colmenas y de producción, esta actividad económica aún debe mejorar la productividad y competitividad de la cadena, para así aprovechar los productos generados por las abejas (miel, propóleos, jalea) que son de interés para el mercado nacional e internacional, considerando a la actual megatendencia de alimentos naturales y sostenibles (Pedraza, 2019; Sánchez et al., 2013).

Por este motivo, la presente investigación tiene como propósito caracterizar con la mayor precisión posible los factores microbiológicos y sensoriales de la miel de abejas producida y comercializada por una empresa de la cadena apícola del departamento de Sucre, mediante la utilización de herramientas y técnicas de analítica de datos, además del uso de software estadístico de acceso libre, con el fin de contribuir a la formación de competencias investigativas, científicas y tecnológicas para el fortalecimiento del sector productivo en la región. En este sentido, por medio de las herramientas y técnicas de analítica de datos, se espera ofrecer de forma directa resultados que puedan contribuir a la mejora de los procesos de dicha empresa, así como a la determinación de los lotes de miel óptimos, de acuerdo a sus características, para que estos puedan servir de base para la realización de otros productos saludables y sostenibles ambientalmente. De esta forma, indirectamente se plantea un aporte metodológico piloto que puede ser ampliado y utilizado por otros productores para visibilizar el potencial apícola del departamento y mejorar los procesos de la cadena productiva del sector.

\section{MATERIALES Y MÉTODOS}

\section{Tipo de investigación}

Este estudio es de tipo descriptivo con un enfoque cuantitativo.

\section{Materia prima}

La miel de abejas objeto de estudio fue recolectada en el departamento de Sucre, en la vereda Flor del Monte perteneciente al municipio de Ovejas, lote 19MC20362 (subregión Montes de María), durante el mes de diciembre de 2019 (19/12/2019); en San Marcos, lote 20MP21110 (subregión San Jorge), durante el mes de abril de 2020 (20/04/2020); y en La Unión, lote 20 MP2174 (subregión San Jorge), durante el mes de marzo de 2020 (15/03/2020). Estas fechas de extracción se deben 
a la recolección planificada por la empresa durante estas épocas, que coinciden con el tiempo de floración de los árboles de santa cruz para el caso del municipio de Ovejas, la cual se da para los meses de noviembre y diciembre, mientras que para el caso de los municipios de San Marcos y La Unión la floración de los árboles de capucho y peralejo se da para los meses de febrero y marzo.

\section{Variables de estudio}

Las variables incluidas en el presente estudio que conforman el factor microbiológico son: esporas de Clostridium sulfito reductor, mohos y levaduras. En el factor sensorial, por su parte, se evalúan los siguientes aspectos: apariencia, textura, olor, color y sabor.

\section{Procesamiento y análisis de la información}

Para desarrollar e implementar un modelo analítico de datos, en primer lugar se deben importar los datos a analizar, para prepararlos, explorarlos y limpiar los datos faltantes. Seguido, se procede a escoger, validar, evaluar y aplicar el o los modelos estadísticos que mejor se ajustan al objetivo o fin último que se requiere, para así poder generar conclusiones, reportes e informes del análisis realizado (Lozano \& Suaterna, 2019; Lozares \& López, 2013). En este estudio, se proyectó que el análisis de datos se centrara en un proceso descriptivo orientado a dar respuesta a lo que sucedió o está sucediendo con la temática en cuestión (Acosta \& Vázquez, 2019; Leal \& Obando, 2020; Lugo \& López, 2018; Quintero et al., 2018). En el caso de la presente aplicación, primero se realizó la revisión bibliográfica de las características teóricas de la analítica de datos. Posteriormente, la información recolectada en los análisis microbiológicos y sensoriales fue codificada, tabulada y analizada para evaluar la composición de las muestras de miel de abejas, utilizando los métodos que se presentan en la siguiente sección.

\section{Métodos}

\section{Análisis microbiológico}

Es un conjunto de operaciones que consisten en una inspección de alimentos por medio de pruebas que permiten determinar los microorganismos presentes en una muestra. A partir de los resultados obtenidos, se puede establecer si un alimento es apto o no para el consumo humano. Los métodos llevados a cabo para los análisis microbiológicos por el laboratorio a las muestras de miel de abejas de los tres apiarios fueron: con respecto a las esporas de Clostridium, sulfito reductor, recuento en placa A.O.A.C., edición 21:2019 (método 972.45); para mohos y levaduras, recuento en placa ISO 21527-1:2008 e ISO 21527-2:2008. Para la realización de los análisis sensoriales por laboratorio a las muestras de miel, con respecto a la apariencia, textura, olor, color y sabor, se realizó el test hedónico ISO 13299-2016.

\section{Análisis sensorial}

La evaluación sensorial permite establecer la calidad de los productos sobre la base de sus atributos (olor, color, textura, gusto, aroma) y distinguir el origen botánico de la miel de abejas, así como defectos de fermentación e impurezas (Mondino \& Ferrato, 2006; Montenegro et al., 2008; Ramírez, 2012). En primera instancia, se presenta el análisis sensorial realizado en laboratorio con personal capacitado en el tema, para el cual se tomaron en cuenta dos muestras de cada uno de los apiarios objeto de estudio. 
A continuación, se presenta el análisis sensorial realizado a consumidores, para el cual se planteó una prueba hedónica, a fin de evaluar sus preferencias sensoriales frente a las tres muestras de miel de abejas con respecto a las características de color, olor, sabor y viscosidad, de acuerdo a lo establecido en GTC 293 de 2018 del Icontec. Las muestras fueron servidas en copas plásticas de media onza y rotuladas con un código de tres dígitos al azar para evitar errores de ordenamiento. Se diseñó y puso en práctica un formulario electrónico con lectura de código QR para la recolección y tabulación de la información (tabla 1).

Para evitar el error de contraste al momento de probar las muestras por parte de los consumidores, se realizó una tabla de distribución de frecuencia, que indica la forma en la que se pidió a los consumidores que probaran las muestras.

Tabla 1. Información prueba hedónica

\begin{tabular}{|c|l|}
\hline Código de las muestras & \multicolumn{1}{c|}{ Información de trazabilidad de la muestra } \\
\hline \multirow{3}{*}{129} & $\begin{array}{l}\text { Muestra de miel de abejas correspondiente al lote 19MC20362. } \\
\text { Color: ámbar extra claro } \\
\text { Olor: floral pesado } \\
\text { Aroma: floral pesado } \\
\text { Textura: viscosa } \\
\text { Sabor: dulce intenso }\end{array}$ \\
\hline \multirow{5}{*}{$\mathbf{4 6 2}$} & $\begin{array}{l}\text { Muestra de miel de abejas correspondiente al lote 20MP21110 } \\
\text { Color: ámbar claro } \\
\text { Olor: floral sutil } \\
\text { Aroma: floral sutil y aromático cítrico } \\
\text { Textura: viscosa } \\
\text { Sabor: dulce intenso }\end{array}$ \\
\hline \multirow{5}{*}{$\mathbf{5 8 1}$} & $\begin{array}{l}\text { Muestra de miel de abejas correspondiente al lote 20MP2174 } \\
\text { Color: ámbar oscuro } \\
\text { Olor: aromático balsámico } \\
\text { Aroma: aromático balsámico } \\
\text { Textura: viscosa } \\
\text { Sabor: dulce de intensidad media }\end{array}$ \\
\hline
\end{tabular}

Fuente: elaboración propia.

\section{Sitio de recolección de las muestras}

La empresa Apiarios de La Sabana SAS tiene ubicados tres apiarios en el departamento de Sucre, el primero en la vereda Flor del Monte, perteneciente al municipio de Ovejas, subregión de Montes de María, la cual posee gran biodiversidad de flora gracias a sus bosques secos tropicales, manglares, lagunas y aguas subterráneas (Aguilera, 2013). Las mieles recogidas en este sector presentan humedad de $18 \%$. En cuanto a la floración, es predominante la del árbol santa cruz. Los otros dos apiarios se encuentran en los municipios de San Marcos y La Unión, pertenecientes a la subregión del San Jorge, la cual cuenta con variedad de flora debido a la presencia de bosques tropicales húmedos, secos y muy secos y sus sabanas naturales, además de que allí se asienta la cuenca del río San Jorge (Sánchez, 2013). Las mieles recogidas en San Marcos y La Unión presentan humedad dl $19 \%$, y la floración predominante es de capucho y peralejo, 
respectivamente. En la figura 1 se puede apreciar la ubicación geográfica de las respectivas subregiones del departamento.

Figura 1. Mapa de las regiones fisiográficas del departamento de Sucre, Colombia

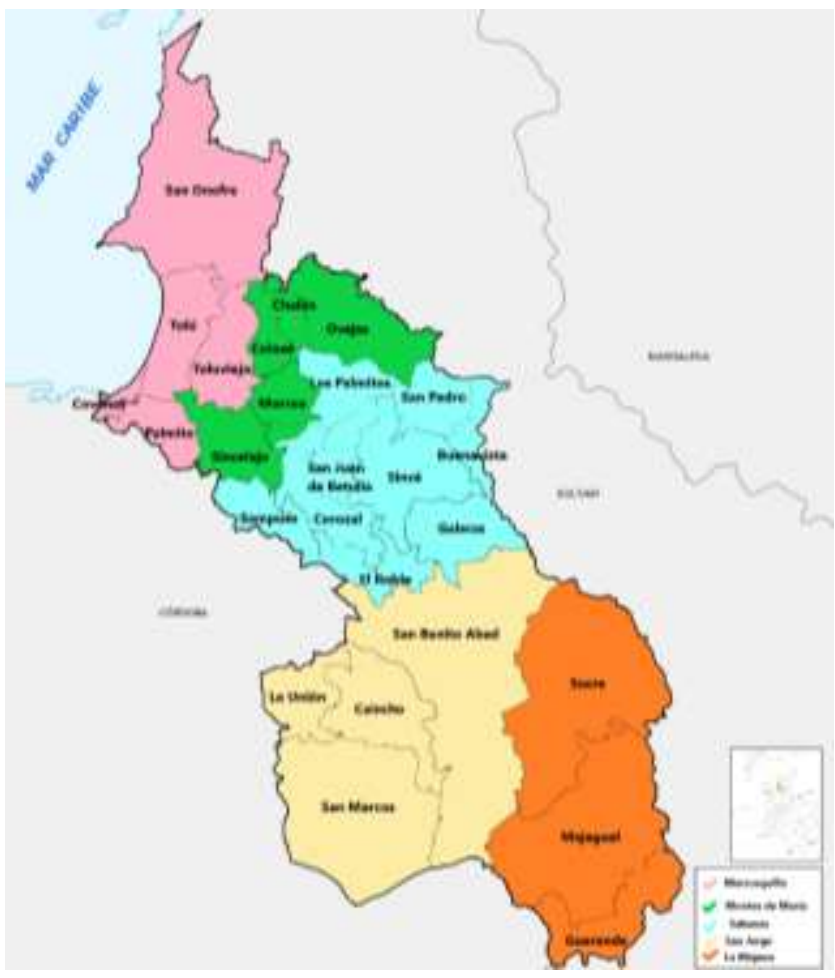

Fuente: elaboración propia a partir de información del Instituto Geográfico Agustín Codazzi (IGAC, 2010).

\section{Análisis estadístico}

La analítica de datos cuenta con diversas herramientas de gran utilidad al momento de caracterizar la información, tales como el análisis multivariado de datos, el cual permite medir más de una característica en un individuo, teniendo como objeto la descripción y exploración de la información (Alcázar, 2001; Avilez et al., 2010; Cattell, 1966; Quintero et al., 2018). Por ello, los datos obtenidos para todas las variables cuantificadas se someten a técnicas de análisis multivariado, tales como el análisis de correspondencia múltiple (ACM) (Castro et al., 2012; Sánchez, 2013; Vertel et al., 2016), entre otras, que permiten evaluar la existencia de patrones de datos y encontrar relaciones entre los parámetros microbiológicos, sensoriales y de origen botánico de la miel, así como defectos de fermentación, presencia de elementos patógenos, grados de contaminación e impurezas.

Este tipo de análisis permite describir y evaluar el sistema de producción de la miel de abejas por medio de las características químicas, microbiológicas y sensoriales de los compuestos presentes en esta, a fin de determinar si es apta o no para su posterior procesamiento y consumo en humanos. 
Los análisis estadísticos para la información recolectada se procesan en el software R ( $R$ Development Core Team, 2008) utilizando los paquetes ADE-4 (Chessel et al., 2004; Husson \& Pages, 2010; Thioulouse, 1997) y FactoClass (Pardo \& del-Campo, 2007).

\section{RESULTADOS}

\section{Los datos}

Para las seis muestras de miel de abejas de los tres apiarios objeto de estudio, los factores microbiológico y sensorial vienen dados por algunas variables cualitativas, las cuales se presentan en las tablas 2 y 3 .

Tabla 2. Datos cualitativos: factor microbiológico

\begin{tabular}{|c|c|c|}
\hline Es.Clo.Sul.R & Moh & Lev \\
\hline$<10$ & $<10$ & $<10$ \\
\hline$<10$ & $<10$ & $<10$ \\
\hline$<10$ & $<10$ & $<10$ \\
\hline$<10$ & $<10$ & $<10$ \\
\hline$<10$ & $<10$ & $<10$ \\
\hline$<10$ & $<10$ & $<10$ \\
\hline
\end{tabular}

Es.Clo.Sul.R: esporas de Clostridium sulfito-reductor ; Moh: moho; Lev: levaduras.

Fuente: elaboración propia.

Tabla 3. Datos cualitativos: factor sensorial

\begin{tabular}{|c|c|c|c|c|}
\hline Apariencia & Textura & Olor & Color & Sabor \\
\hline Translúcido con brillo & Viscosidad media-alta & Floral con notas típicas & $\begin{array}{c}\text { Ámbar } \\
\text { oscuro brillante }\end{array}$ & Dulces en media intensidad \\
\hline Translúcido con brillo & Viscosidad media-alta & Floral con notas típicas & $\begin{array}{c}\text { Ámbar oscuro } \\
\text { brillante }\end{array}$ & Dulces en media intensidad \\
\hline Translúcido con brillo & Viscosidad media & Notas típicas de miel & Ámbar claro medio & $\begin{array}{c}\text { Dulces en baja-media } \\
\text { intensidad }\end{array}$ \\
\hline Translúcido con brillo & Viscosidad media & $\begin{array}{c}\text { Notas típicas de } \\
\text { miel }\end{array}$ & $\begin{array}{c}\text { Ámbar claro } \\
\text { brillante }\end{array}$ & $\begin{array}{c}\text { Dulces en media-baja } \\
\text { intensidad }\end{array}$ \\
\hline Translúcido con brillo & Viscosa & Notas medias dulces & $\begin{array}{c}\text { Ámbar claro bril- } \\
\text { lante }\end{array}$ & $\begin{array}{c}\text { Dulces en alta intensidad } \\
\text { Translúcido con brillo }\end{array}$ \\
\hline
\end{tabular}

Fuente: elaboración propia.

\section{Procesamiento y tratamiento de tablas de datos cualitativos}

Para el análisis estadístico de datos categóricos en el software $\mathrm{R}$, estos deben pasar primero por un proceso de tratamiento y depuración, para lo cual deberán cumplir las siguientes especificaciones:

(i) La tabla de datos debe estar en formato .txt (delimitado por tabulaciones). 
(ii) En el caso de que un individuo o unidad experimental no cuente con información de alguna de las variables evaluadas, este deberá ser retirado del estudio.

(iii) Se deben codificar las categorías de las variables con el fin de facilitar la visualización de los resultados, para lo cual se recomienda simplificar las palabras con una extensión máxima de ocho caracteres, evitar la utilización de números al empezar la palabra codificada, evitar el uso de tildes y la letra $\tilde{n}$. Con estas especificaciones realizadas a la tabla de datos, se exporta al software.

\section{Análisis univariado}

\section{Factor microbiológico}

Para este factor, el recuento de esporas, levaduras y mohos en las muestras de miel de abejas permite evidenciar que el nivel es igual para todos los lotes incluidos en el análisis (tabla 4).

Tabla 4. Número de ensayo vs. nivel de esporas, levaduras y mohos

\begin{tabular}{|c|c|c|c|c|c|c|}
\hline \multicolumn{7}{|c|}{ N.o de ensayos } \\
\hline & $\mathbf{2 1 6 7 8}$ & $\mathbf{2 1 6 7 9}$ & $\mathbf{2 1 6 8 0}$ & $\mathbf{2 1 6 8 1}$ & $\mathbf{2 1 6 8 2}$ & $\mathbf{2 1 6 8 3}$ \\
\hline Nivel de esporas <10 & 1 & 1 & 1 & 1 & 1 & 1 \\
\hline Nivel de levaduras <10 & 1 & 1 & 1 & 1 & 1 & 1 \\
\hline Nivel de mohos <10 & 1 & 1 & 1 & 1 & 1 & 1 \\
\hline
\end{tabular}

Fuente: elaboración propia.

\section{Factor sensorial}

Con respecto al color, se evidencia que el preferido por las personas participantes en el análisis sensorial fue el de la muestra $\mathrm{M} 1$, correspondiente al código 129 del Lote 19MC20362, del apiario ubicado en Flor del Monte (Ovejas). El $76 \%$ de los que seleccionaron esta muestra como preferida la ubicaron en la categoría "Mucho", mientras que en términos del color la de menor preferencia fue la muestra M3 (58,3\%), con el código 581 del Lote 20MP2174, proveniente del apiario ubicado en el municipio de La Unión. Es de resaltar que la muestra M1 presenta un color extra claro, según la evaluación realizada por expertos (Smart Bee Ciencia y Tecnología), mientras que la muestra M3 presenta un color ámbar oscuro.

En el caso de la viscosidad, se puede evidenciar mayor preferencia por la muestra M1 (51\%), además de que el $76 \%$ de los que seleccionaron esta muestra como preferida la ubicaron en la categoría "Mucho". La muestra menos preferida en términos de viscosidad fue M3 (54,7\%). Según la evaluación realizada por expertos (Smart Bee Ciencia y Tecnología), las muestras M1 y M2 presentan una consistencia viscosa y no se descarta cristalización durante el almacenamiento.

Finalmente, para el olor se reporta preferencia por la muestra M1 $(48,4 \%)$, ya que $81,3 \%$ de los que seleccionaron esta muestra como preferida la posicionaron en la categoría "Mucho", mientras que la muestra menos preferida en 
términos del olor fue M3 (61,5\%). Según la evaluación realizada por expertos (Smart Bee Ciencia y Tecnología), la muestra M1 presenta un olor característico de la familia de olores florales, subfamilia floral pesado, con notas aromáticas cítricas y de fruta procesada de intensidad alta, mientras que la M3 presenta un olor característico de la familia de olores aromáticos, subfamilia balsámico y resinoso de intensidad media.

\section{Análisis bivariado de las variables incluidas}

\section{Color M1}

Para los individuos que seleccionaron como color preferido el de la muestra M1, se evidencia que el $71 \%$ a su vez prefirió la viscosidad de la misma muestra, el $64 \%$ su olor y el $68 \%$ su sabor. Le siguió en elección la M2, dado que de los individuos que seleccionaron el color de la muestra M1, el $21 \%$ prefirió la viscosidad de la muestra $\mathrm{M} 2$, el $30 \%$ el olor de la $\mathrm{M} 2$ y el $31 \%$ el sabor de la M2. Por último estuvo la M3, puesto que $8 \%$ de los individuos que seleccionaron el color de la muestra M1 prefirió la viscosidad de la muestra M3, el $6 \%$ el olor de la M3 y el $1 \%$ el sabor de la M3. Asimismo, se puede evidenciar que las asociaciones entre el color y las demás variables (viscosidad, olor y sabor) son estadísticamente significativas, teniendo en cuenta los $p$-valores $(<0,05)$ de las pruebas Chi-cuadrado realizadas. Por esto, la mayoría de los individuos que seleccionaron como preferido el color de la muestra M1 (código 129 del Lote 19MC20362, apiario ubicado en Flor del Monte, Ovejas) seleccionaron también como características preferidas de viscosidad, olor y sabor las correspondientes a la misma muestra.

\section{Color M2}

Para los individuos que seleccionaron como color preferido el de la muestra M2 (57 personas), el 56,14\% prefirió además la viscosidad de la misma muestra, el 52,62 \% su olor y el $66,66 \%$ su sabor. Siguió en elección la M1, de modo que $29,82 \%$ de los individuos que seleccionaron el color de la muestra M2 prefirieron la viscosidad de la muestra M1, 31,57\% el olor de la M1 y 24,56\% el sabor de la M1. Por último estuvo la M3, dado que de los individuos que seleccionaron el color de la muestra M2, el 14,03\% prefirieron la viscosidad de la muestra M3, el $15,78 \%$ el olor de la M3 y el 8,77 \% el sabor de la M3. Se puede evidenciar así que las asociaciones entre el color y las demás variables (viscosidad, olor y sabor) son estadísticamente significativas, teniendo en cuenta los $p$-valores $(<0,05)$ de las pruebas Chicuadrado realizadas. Por esta razón, la mayoría de los individuos que seleccionaron como preferido el color de la muestra M2 (código 462 del Lote 20MP21110, apiario ubicado en San Marcos, Sucre) seleccionaron también como características preferidas de viscosidad, olor y sabor las correspondientes a la misma muestra.

\section{Color M3}

Para quienes seleccionaron como color preferido el de la muestra M3, se evidencia que $60 \%$ de estos prefirieron también la viscosidad de la misma muestra, el $51,42 \%$ su olor y el $1 \%$ su sabor. Le siguió en elección la M1. De los individuos que seleccionaron el color de la muestra M3, el 28,57 \% prefirió la viscosidad de la muestra M1, el 31,42\% el olor de la M1 y el $68 \%$ el sabor de la M1. Finalmente aparece la M2, ya que $11,42 \%$ de los individuos que seleccionaron el color de la muestra M3 prefirieron la viscosidad de la muestra M2, 17,14\% su olor y $31 \%$ su sabor. También se puede evidenciar que las asociaciones entre el color y las demás variables (viscosidad, olor y sabor) son estadísticamente significativas, teniendo en cuenta los $p$-valores $(<0,05)$ de las pruebas Chi-cuadrado realizadas. Por lo ante- 
Menco-Tovar, Aı, Méndez-Ramos, M., Cáceres-Pestana, K. y Vertel-Morinson, M. (2022), httpš//doi..org/10.21789/22561498.1768

rior, la mayoría de los individuos que seleccionaron como preferido el color de la muestra M3 (código 581 del Lote 20MP2174, apiario ubicado en La Unión, Sucre) seleccionaron también como características preferidas de viscosidad y olor las correspondientes a la misma muestra, mientras que para el caso del sabor en su mayoría prefirieron la muestra M1.

\section{Análisis multivariado. Análisis de correspondencia múltiple (ACM)}

\section{Análisis sensorial en laboratorios}

Los valores propios del ACM dan cuenta de que el segundo componente principal recoge el $88,32 \%$ de la variabilidad inicial, lo cual es un valor aceptable a nivel estadístico, que refleja la calidad de la representatividad de los datos. En ese sentido, el análisis correspondiente al ACM se realizará para las dos primeras dimensiones en plano factorial, atendiendo los valores propios identificados, como se puede constatar en la tabla 5 y la figura 2 .

Tabla 5. Eigenvalue ACM: factor sensorial

\begin{tabular}{|c|c|c|c|}
\hline & Eigenvalue variance & Percent cumulative & Variance percent \\
\hline Dim.1 & $5,430223 \mathrm{e}-01$ & $4,791373 \mathrm{e}+01$ & 47,91373 \\
\hline Dim.2 & $4,579650 \mathrm{e}-01$ & $4,040867 \mathrm{e}+01$ & 88,32240 \\
\hline Dim.3 & $1,090346 \mathrm{e}-01$ & $9,620700 \mathrm{e}+00$ & 97,94310 \\
\hline Dim.4 & $2,331153 \mathrm{e}-02$ & $2,056900 \mathrm{e}+00$ & 100,00000 \\
\hline Dim.5 & $1,196302 \mathrm{e}-32$ & $1,055560 \mathrm{e}-30$ & 100,00000 \\
\hline
\end{tabular}

Fuente: elaboración propia.

Figura 2. ACM. Contribuciones de las variables-individuos: factor sensorial

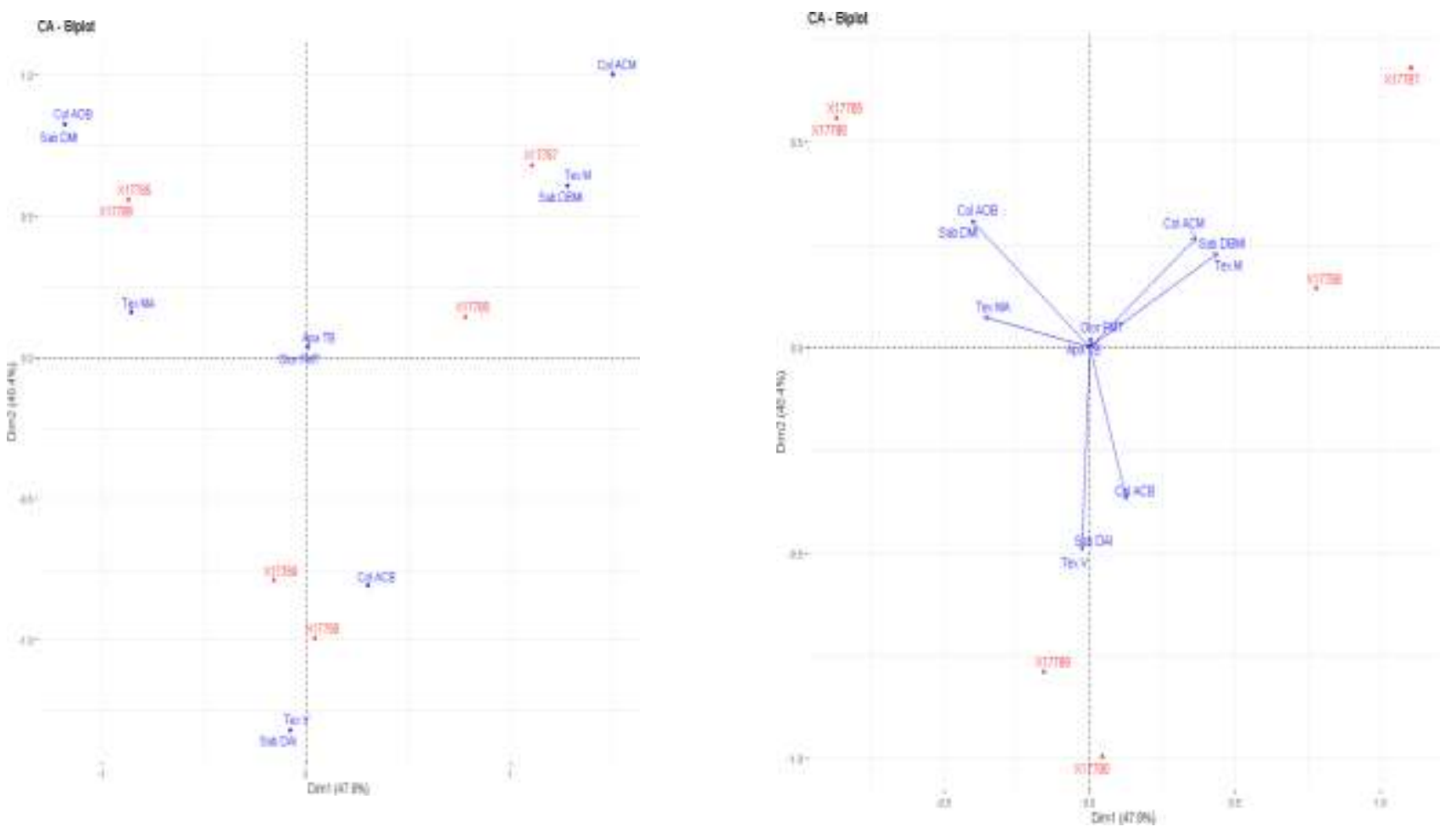

Fuente: elaboración propia. 
A la hora de explicar el comportamiento de las variables sensoriales de las muestras de miel, se evidencia que las que más contribuyen a las dimensiones son color, sabor y textura. Así, teniendo en cuenta que desde el punto de vista estadístico estas variables son las que mayor contribución generan al análisis de la miel de abejas, se recomienda tenerlas presentes en futuros procesos.

En términos de los factores sensoriales, se puede establecer que las muestras SP664 y SP668, tomadas del municipio de la Unión, presentan condiciones similares: la apariencia es traslúcida con brillo, en la parte superior se observa liso y en la parte inferior se observan pequeñas burbujas, presenta textura con viscosidad media-alta, olor floral con notas típicas del producto, color ámbar oscuro brillante y sabor típico de la miel con notas dulces de media intensidad.

Las muestras SP665 y SP669, del municipio de Flor del Monte, presentan también condiciones similares: apariencia traslúcida con brillo, liso en la parte superior y con pequeñas burbujas en la parte inferior, textura de viscosidad media, olor floral con notas típicas del producto, color ámbar claro brillante y sabor típico de la miel con notas dulces de intensidad baja-media. Por último, las muestras SP666 y SP670 del municipio de San Marcos presentan similares condiciones: apariencia traslúcida con brillo, liso en la parte superior y con pequeñas burbujas en la parte inferior, textura viscosa sin nivel de clasificación, olor floral con notas típicas del producto, color ámbar claro brillante y sabor típico de la miel con notas dulces altas.

\section{ACM análisis sensorial en catadores no expertos}

Teniendo en cuenta los ACM realizados para color, viscosidad, olor y sabor (figuras 3-5) se evidencia:

(i) Los que prefirieron el color, viscosidad, olor y sabor de la muestra M1 son mayormente personas a quienes no les gustó el color, viscosidad, olor y sabor de la M2, que compran y consumen frecuentemente miel de abejas, pero que nunca han participado en un análisis sensorial, del área urbana, de estratos socioeconómicos 2 y 3 , con ingresos entre COP 0 y 500.000, en la edad E4, que corresponde a personas mayores de 40 años, presentan hipertensión y sobrepeso, usan la miel de abejas para fines medicinales y la consumen en su mayoría como snack, en las meriendas y algunos en la cena.

(ii) Quienes prefirieron el color, viscosidad, olor y sabor de la muestra M2 mayormente no les gustó el color, viscosidad, olor y sabor de la M3; son personas que compran esporádicamente miel de abejas, aseguran tal vez haber participado en análisis sensoriales con anterioridad, son de estratos 1 y 4 , algunos sin ingresos y otros con ingresos entre COP 1.000 .000 y 2.000 .000 (o más), casados, otros solteros, con edades entre 18-25 años y 26-30 años, quienes usan la miel para varios procesos y la consumen en su mayoría en el almuerzo. Según esto, la M2 fue seleccionada como preferida por personas jóvenes, de estratos altos, que reciben ingresos altos.

(iii) Por último, los individuos que prefirieron la M3 en cuanto al color, viscosidad, olor y sabor son mayormente de género masculino, no prefirieron la $\mathrm{M} 1$, con edades entre 31-40 años, con ingresos entre COP 600.000 y 1.000 .000 , de estrato 2 , quienes viven en unión libre. Esta muestra fue seleccionada por personas de género masculino, 
pero que afirman usarla y consumirla, con edad e ingresos promedios.

(iv) Las variables que están aportando en mayor medida a la construcción de las dos dimensiones correspondientes al caso del color, viscosidad, olor y sabor son la ubicación geográfica, el estado civil y la presencia de alguna enfermedad.

Figura 3. Análisis de correspondencia múltiple: color

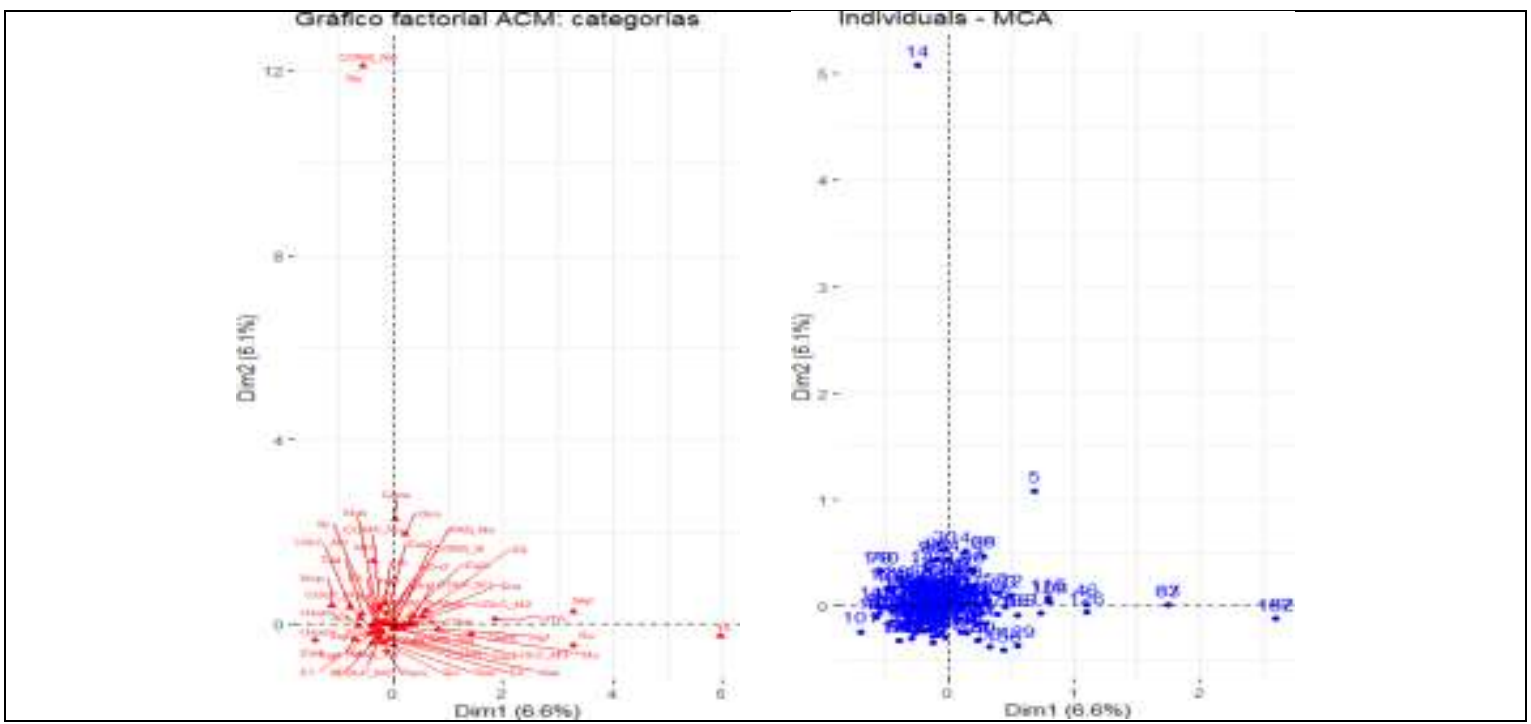

Fuente: elaboración propia.

Figura 4. Análisis de correspondencia múltiple: viscosidad
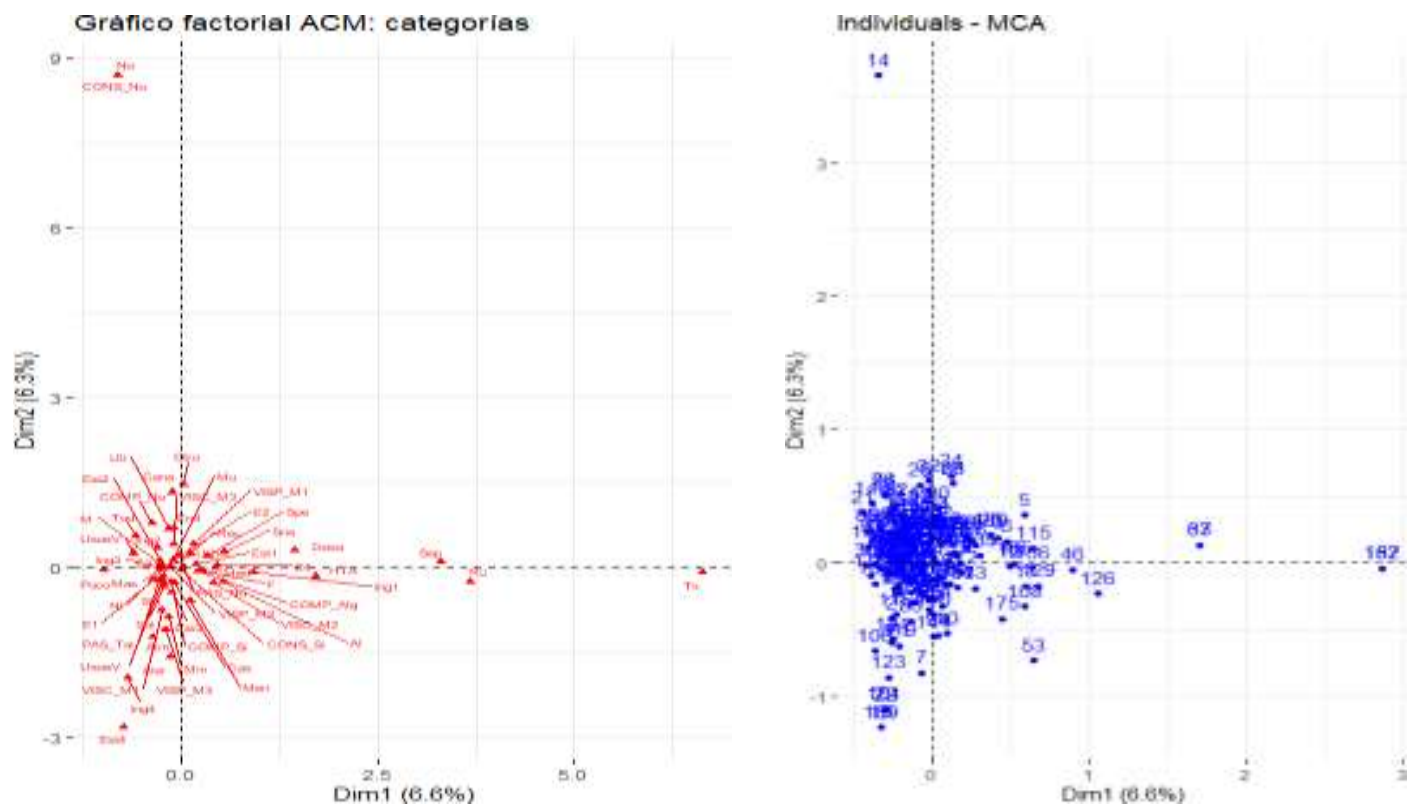

Fuente: elaboración propia. 
Figura 5. Análisis de correspondencia múltiple: olor
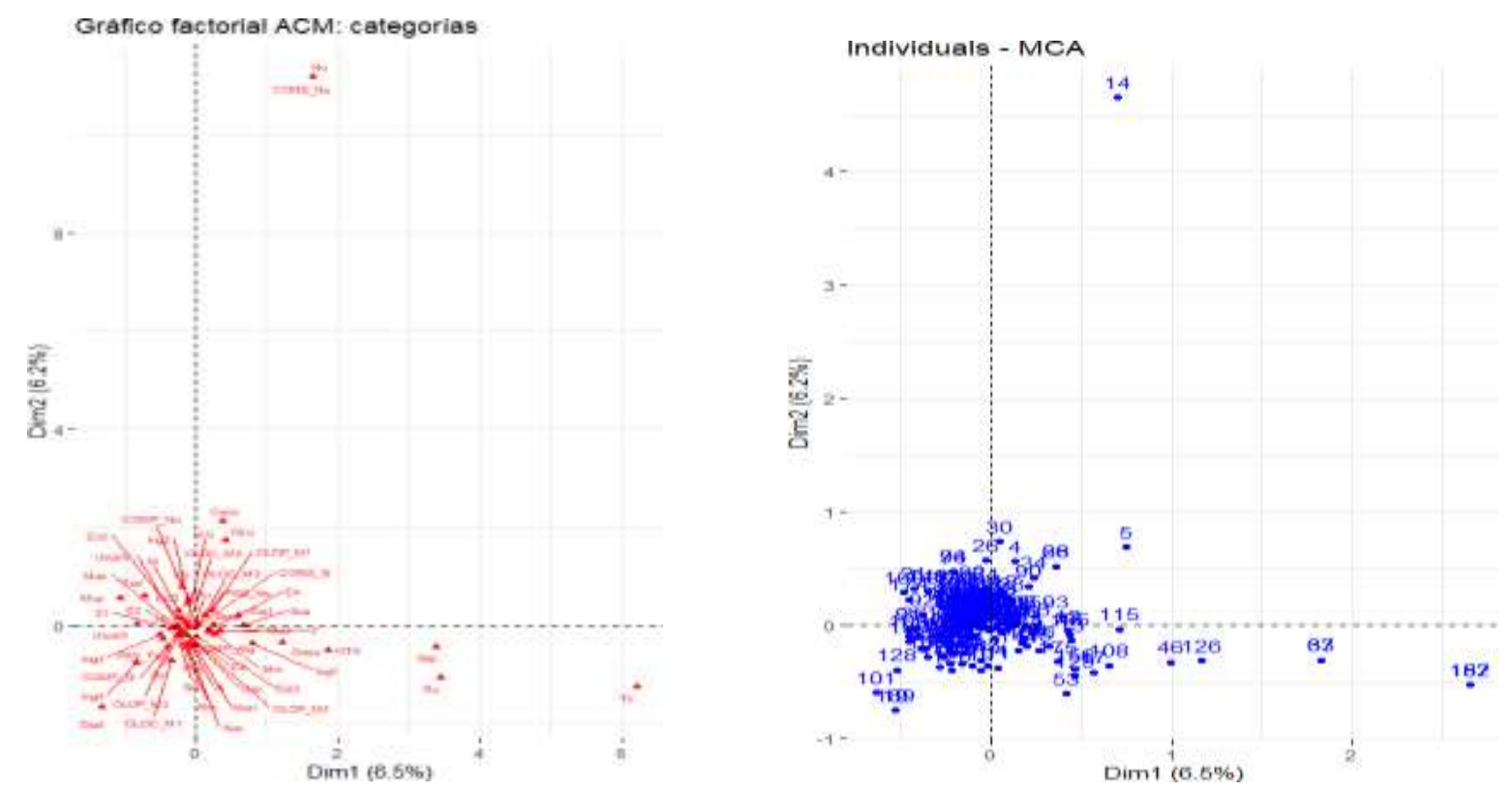

Fuente: elaboración propia.

\section{DISCUSIÓN}

En Colombia, el Ministerio de Agricultura y Desarrollo Rural reconoce a la organización de la cadena productiva apícola a través de la Resolución 282 de 2012, lo cual significa una mayor responsabilidad con el desarrollo competitivo de cada uno de los eslabones que componen la cadena. Adicionalmente, el Ministerio de la Protección Social de Colombia publicó en 2010 los requisitos fisicoquímicos y microbiológicos de la miel de abejas dentro de la Resolución 00001057, en la cual se establece el reglamento técnico sobre los requerimientos sanitarios que debe cumplir la miel para consumo humano en el país; aunque actualmente no está vigente, esta se encuentra estrechamente ligada y contenida en la Norma Técnica Colombiana 1273.

Para el caso de la presente investigación, las muestras de miel evaluadas en el ámbito microbiológico presentan un comportamiento acorde con lo establecido por las normas mencionadas. Estos resultados además guardan relación con algunos estudios realizados en departamentos como Magdalena y Antioquia, en los que las muestras de miel evaluadas presentan parámetros microbiológicos que también indican que el producto derivado de esas zonas es apto para el consumo humano. Asimismo, estos estudios resaltan que la miel de Apis mellifera en zonas con variedad de flora y ambiente tienden a contener elevados niveles de azúcar y baja humedad, que ayudan a contrarrestar la aparición de microrganismos. Por esto, es posible concluir que las condiciones de biodiversidad de las zonas en el departamento de Sucre sean las causantes de que los parámetros microbiológicos de las mieles extraídas en estas áreas, dadas sus características, presenten niveles óptimos con poca contaminación microbiológica. Estos resultados, según otros autores, son de vital importancia en el proceso de almacenamiento del producto, debido a que una baja carga microbiana conlleva a que la miel tenga una vida útil más larga y una mayor aceptación (Hernández et al., 2014). 
En términos sensoriales, se evidenciaron resultados muy parecidos a los encontrados por investigaciones en el Suroeste Antioqueño, donde dichas mieles se caracterizan por ser de color ámbar, aroma agradable típico del producto, textura fina y sabor dulce intenso (Ospina, 2014). Además, entre más claro el color y más intenso el dulce de las mieles, mayor grado de aceptabilidad entre los catadores, tanto expertos como no expertos.

Aunque a nivel nacional existe un gran auge por el desarrollo de la apicultura como actividad económica, son muy pocas las investigaciones que se realizan en términos microbiológicos y sensoriales a nivel empresarial sobre la miel de abejas, por lo cual se resalta la importancia de vincular herramientas de desarrollo tecnológico que permitan fortalecer los procesos productivos de la mano de la ciencia, tecnología e innovación, sobre todo en la pequeñas y medianas empresas.

Por su parte, en relación a la analítica de datos, son casi nulas las aplicaciones realizadas en el área apícola. Sobre ello, es importante resaltar que las técnicas de la analítica de datos permiten la realización de estudios del comportamiento simultáneo de varias variables, resumiendo y sintetizando la información contenida en el conjunto de datos, para así finalmente lograr una mayor comprensión del fenómeno en estudio y obtener una visión más amplia de la realidad (Robaina et al., 2001; Vargas et al., 2013). Estas constituyen una herramienta poderosa para analizar los datos en términos de muchas variables, permitiendo extraer la máxima información, a partir de lo cual se pueden caracterizar e identificar problemáticas y necesidades que las empresas pueden tratar para mejorar sus procesos a nivel de cualquier área de su cadena productiva (Avilés et al., 2010).

En la actualidad existen paquetes estadísticos tales como el software $R$ ( $R$ Development Core Team, 2008), un lenguaje de programación estadístico libre, gratis y de código abierto que ofrece una gama de posibilidades para conectar datos, leerlos, procesarlos y analizarlos, por medio de técnicas clásicas y avanzadas que permiten el manejo de la información. Este software está siendo mayormente utilizado por empresas y organizaciones para la enseñanza y la investigación estadística, debido a su robustez y las infinitas posibilidades que ofrece para el manejo de datos, además de que cuenta con gran aceptación en el campo investigativo, lo cual ofrece un soporte de confiabilidad a los resultados obtenidos en las investigaciones.

\section{CONCLUSIÓN}

Las mieles estudiadas presentan límites permitidos en cuanto a los criterios establecidos para el factor microbiológico, lo cual indica que se trata de mieles maduras, libres de patógenos microbianos o toxinas (Ferrer \& Morales, 2005). Por otra parte, en cuanto a los análisis sensoriales realizados por laboratorio y por catadores, no se notan diferencias significativas. Se constata que entre más claro el color y dulce intenso de las mieles, mayor grado de aceptabilidad, además de que se trata de mieles homogéneas, que no presentan impurezas. Las muestras SP666 y SP670 tomadas en el municipio de San Marcos presentan condiciones similares a todas las demás (apariencia traslúcida con brillo, liso en la parte superior y con pequeñas burbujas en la parte inferior, textura viscosa sin llegar a nivel de clasificación, olor floral con notas típicas del producto, color ámbar claro brillante), con la diferencia que estas presentan sabor típico de miel con notas dulces en nivel alto, mientras que el resto presenta notas dulces en nivel medio y medio bajo. 
Los resultados obtenidos de la presente investigación fueron utilizados por una empresa de la cadena apícola del departamento de Sucre para determinar las características de los lotes de miel que pueden servir de base para la realización de otros productos saludables a base de miel de abejas. Por medio de la articulación de los procesos productivos y la investigación científica, específicamente en el área de la analítica de datos, esta aplicación proporciona un aporte metodológico que puede ser replicado a mayor escala en la cadena apícola del departamento, concientizando e incentivando así la realización de estudios e investigaciones sobre la calidad de la miel de abejas producida y comercializada a nivel de las pequeñas y medianas empresas en el departamento.

\section{REFERENCIAS}

Acosta, D., \& Vásquez, S. (2019). Analítica de datos para el soporte en la toma de decisiones en el área de distribución y ventas de la distribuidora Farmacéutica La Libertad S.R.L. utilizando Microsoft Azure y la metodología de Larissa Moss [tesis de grado, Universidad Privada Antenor Orrego]. Repositorio Universidad Privada Antenor Orrego.

Aguilera, M. (2013). Montes de María: Una subregión de economía campesina y empresarial. Documentos de Trabajo sobre Economía Regional, 195, 1-93. https://doi.org/10.32468/dtseru.195

Alcázar, A. (2001). Aplicaciones del análisis multivariante a la diferenciación de tipos de cerveza [tesis de grado, Universidad de Sevilla]. Repositorio Universidad de Sevilla.

Aristizábal, J. (2017). Diseño y aportes de un modelo para minería de datos educativos en aulas de educación media de carácter presencial [tesis doctoral, Universidad Santo Tomás]. Repositorio USTA.

Avilez, J., Escobar, P., Von-Fabeck, G., Villagran, K., Matamoros, R., \& García, A. (2010). Caracterización productiva de explotaciones lecheras empleando metodología de análisis multivariado. Revista Científica, 20(1), 74-80.

Castro, L., Carvajal, Y., \& Ávila, Á. (2012) Análisis clúster como técnica de análisis exploratorio de registros múltiples en datos meteorológicos. Revista EIDENAR, 11, 11-20. McNally.

Cattell, R. (1966). Handbook of multivariate experimental psychology. Rand

Chessel, D., Dufour, A., \& Thioulouse, J. (2004). The ade4 Package - I: Onetable Methods. $R$ News, 4, 5-10.

Dardón, M., \& Enríquez, E. (2008). Caracterización fisicoquímica y antimicrobiana de la miel de nueve especies de abejas sin aguijón (Meliponini) de Guatemala. Interciencia, 33(12), 916-922.

Ferrer, P., \& Morales, M. (2005). Determinación de la calidad microbiológica de la miel [tesis doctoral, Universidad de la Republica Uruguay]. Repositorio Universidad de la Republica Uruguay. 
Gómez, A. (2019). Plan de negocios para la creación de una empresa de Bl y analítica de datos dirigida a las pymes del sector ecommerce en Colombia [tesis de maestría, Universidad EAN]. Repositorio Universidad EAN.

González, A., \& Echenique, R. (2017). Estudio de factibilidad de la creación de un apiario en el municipio de Arjona, Bolivar [tesis de grado, Universidad de Cartagena]. Repositorio Universidad de Cartagena.

Hernández, C., Ascencio, D., \& Quicazán, M. (2014). Indicadores microbiológicos de estabilidad e inocuidad de miel de Mellipona eburnea durante el almacenamiento. Revista Facultad Nacional de Agronomía Medellín, 67(2), 828-830.

Husson, F., \& Pages, J. (2010). Exploratory multivariate analysis by example using R. Taylor and Francis Group. https://doi.org/10.1201/b10345

Instituto Geográfico Agustín Codazzi [IGAC]. (2010). División politicoadministrativa departamento de Sucre [Gráfico]. https://sigot.igac.gov.co/es/mapastematicos/departamentos

Joyanes, A. (2014). Big Data. Análisis de grandes volúmenes de datos en organizaciones. Alfaomega Grupo Editor.

Leal, J., \& Obando, R. (2020). Ruta de competitividad para los destinos turisticos regionales colombianos fundamentada en el MMGO y la analítica de datos [tesis de maestría, Universidad EAN]. Repositorio Universidad EAN.

Lozano, C., \& Suaterna, J. (2019). Analítica de datos para el rendimiento de los cultivos de aguacate hass en Colombia [trabajo de grado, Universidad Externado de Colombia]. Repositorio Universidad Externado de Colombia.

Lozares, C., \& López, P. (2013). El análisis multivariado: definición, criterios y $\begin{array}{lllll}\text { clasificación. Revista de Sociología, 37, } & \text { 9-29. }\end{array}$ https://doi.org/10.5565/rev/papers/v37n0.1594

Lugo, C., \& López, J. (2018). Analítica de datos con aplicación en un caso práctico, mediante el uso de una herramienta libre [tesis de grado, Universidad Tecnológica de Pereira]. Repositorio UTP.

Mondino, M., \& Ferratto, J. (2006). El análisis sensorial, una herramienta para la evaluación de la calidad desde el consumidor. Revista Agromensajes, 18, 16-48.

Montenegro, G., Gómez, M., Pizarro, R., Casaubon, G., \& Peña, R. (2008). Implementación de un panel sensorial para mieles chilenas. Revista Ciencia e Investigación Agraria, 35(1), 51-58. https://doi.org/10.4067/S0718-16202008000100005

Morales, S., Morales, M., Andrade, A., \& Cevallos, L. (2019). Analítica de datos puros dentro del ámbito productivo y reproductivo de las ganaderías de leche. Revista Arbitrada Interdisciplinaria Koinonía, 5(9), 287-310. http://dx.doi.org/10.35381/r.k.v5i9.649

Ordieres, J. (2014). Big Data e loT: claves del modelo de negocio para la empresa industrial del siglo XXI. Economía Industrial, 392, 113-122. 
Organización de las Naciones Unidas para la Alimentación y la Agricultura [FAO]. (2014). Agricultura familiar en América Latina y el Caribe. FAO FIAT PANIS.

Ortiz, M., Aguilar, L., \& Giraldo, L. (2015). Los desafíos de marketing en la era del big data. E-Ciencias de la Información, 6(1), 1-30. http://dx.doi.org/10.15517/eci.v6i1.19005

Ospina, A. (2014). Evaluación de las características de la miel de abejas proveniente del suroeste antioqueño y de las condiciones necesarias para su liofilización [tesis de grado, Universidad Pontificia Bolivariana]. Repositorio UPB.

Pardo, C., \& del-Campo, P. (2007). Combinación de métodos factoriales y de análisis de conglomerados en R: el paquete FactoClass. Revista Colombiana de Estadistica, 30(2), 231-245.

Pastor, X. (2017). Análisis de datos sanitarios y retorno de la inversión para la atención al paciente: desafíos para un hospital universitario. Revista de Bioética y Derecho, 411, 47-56. https://doi.org/10.1344/rbd2017.41.19909

Pedraza, P. (2019). Plan de negocios de la exportación de miel de abejas a Alemania [trabajo de grado, Fundación Universidad de América]. Repositorio Fundación Universidad de América.

Quintero, J., Villanueva, D., \& Gómez, F. (2018). Analítica de datos para sistemas de costos basados en actividades en la era de big data. Revista del Instituto Internacional de Costos, 1, 64-82.

R Development Core Team (2008). R: A language and environment for statistical computing. R Foundation for Statistical Computing.

Ramírez, J. (2012). Análisis sensorial: pruebas orientadas al consumidor. RECITEIA, 12(1), 83-102.

Robaina, G., Pollo, J., Morales, J., \& Robaina, R. (2001). Análisis multivariado de factores de riesgo de prematuridad en Matanzas. Revista Cubana de Obstetricia y Ginecología, 27(1), 62-69.

Sánchez, A. (2013). La economía del bajo San Jorge. Documentos de Trabajo sobre Economía Regional, 189, 1-46.

Sánchez, O., Castañeda, P., Muños, G., \& Tellez. G. (2013). Aportes para el análisis del sector apícola colombiano. Journal of Agricultural Science and Technology, 2(4), 469-483.

Tascón, M. (2013). Introducción: Big Data. Pasado, presente y futuro. Telos: Cuadernos de Comunicación e Innovación, 95, 47-50.

Thioulouse, J., Chessel, D., Doledec, S., \& Olivier, J. (1997). ADE-4: A multivariate analysis and graphical display software. Statistics and Computing, 7, 75-83. https://doi.org/10.1023/A:1018513530268 
Menco-Tovar, A., Méndez-Ramos, M., Cáceres-Pestana, K. y Vertel-Morinson, M. (2022), https://doi.org/10.21789/22561498.1768

Vargas, B., Solís, O., Sáenz, F., \& León, H. (2013). Caracterización de hatos lecheros en Costa Rica mediante análisis multivariado. Revista Agronomía Mesoamericana, 24(2), 257-275.

Vertel, M., Botero, L., \& Cepeda, J. (2016). Análisis multivariado de datos. Aplicación: Sistemas de producción doble propósito. Ediciones Universidad Simón Bolívar. 